$$
\begin{gathered}
\text { NS } 6-426 \\
\text { C.I.t, }
\end{gathered}
$$

\title{
A MODEL OF W VIRGINIS WITH RV TAURI CHARACTERISTICS
}

Non-linear calculation methods (Christy 1964) have been applied to a model intended to represent $W$ Virginis. The original objective was to find if a model with mass near $1.0 \mathrm{M}$ could show the long period of $W$ Virginis, The radius, effective temperature, and luminosity of the static model were chosen with reference to the study of Abt (1954) but $\mathrm{T}_{e}$ and $\mathrm{L}_{\mathrm{o}}$ were adjusted to arrive at instability with approximately the right period. The chosen model had $M=1.75 \times 10^{33} \mathrm{gm}\left(0.88 \mathrm{M}_{\odot}\right)$, $L_{0}=7.0 \times 10^{36} \mathrm{erg} / \mathrm{sec}\left(M_{b}=-3.4\right), R_{0}=3.2 \times 10^{12} \mathrm{~cm}$, and $T_{e}=5500^{\circ} \mathrm{K}$. The composition was given by $\mathrm{Y}=0.45$ and $\mathrm{Z}=0.002$. This led to a period of 18.5 days and a surface gravity of 11.5 . In addition to this model, a few nearby models with varied $\mathrm{T}_{\mathrm{e}}$ were examined for instability.

Although the model was violently unstable at $5500^{\circ} \mathrm{K}$, increasing $\mathrm{T}_{\mathrm{e}}$ to $6000^{\circ} \mathrm{K}$ with the same $\mathrm{L}_{\mathrm{o}}$ gave a model that was stable in the fundamental and in the first harmonic. This model, however, showed an increasing amplitude (instability) in the third harmonic (the only case I have found). The zone structure with 38 zones is not reliable for such a short wavelength and no attempt was made to study this instability with an adequate structure since it would involve at least twice as many zones. Models with other masses than $0.88 \mathrm{M}$ were not investigated with the high Iuminosity needed for a $\mathrm{W}$ Virgints model. Thus the results obtained here in no way indicate that the chosen mass is correct. It is merely a possible value.

\section{N $66-16391$}

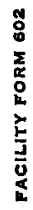

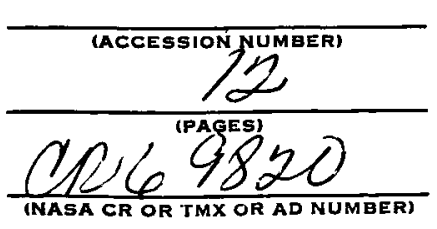

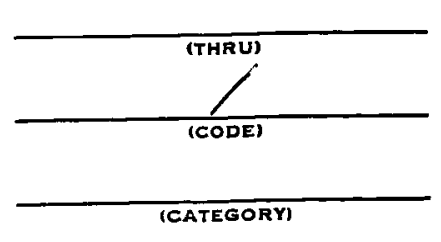

\section{GPO PRICE}

CFSTI PRICE(S) S

Hard copy $(\mathrm{HC}$

Microfiche (MF) 
The model chosen to represent $\mathrm{W}$ Virgin is with $\mathrm{T}_{\mathrm{e}}=5500^{\circ} \mathrm{K}$ showed an exceedingly rapid amplitude growth, increasing from half to full amplitude in about three periods (see Flgure la). This was the most rapid growth (and most violent instability) of any models so far studied, in general, however, rapld growth characterizes unstable models of large luminosity to mass ratio because of their abnormally low density envelopes. Another peculiar feature of this model was the very strong relative driving of the hydrogen Ionization zone. The work developed in the hydrogen zone per period was 4.4 percent of the total radiated flux whereas it was only 2.8 percent in the helium + ionization zone. When the motion was fully developed, the pulsation showed a very large amplitude with some irregularity which then settled down to a fairly regular modulation of the fundamental in which alternate periods had larger and smaller amplitude (Figure 2).

The period found (about 18.5 days) was remarkably long. The period quoted here is the fundamental period of the small amplitude oscillation which is the meaningful quantity in pulsation theory. It is not the repetition period of the motion which was twice as long. The pulsation constant $Q=P \sqrt{\rho / P_{\odot}}=0.0560$ compared to values near 0.038 in most of my RR Iyrae models (Christy 1966). Calculations of Schwarzschild (1941) Indicate that the ratio of first harmonic to fundamental period is reduced to near 0.6 for such a large value of $Q$, whereas the ratio is near 0.75 in the RR Lyrae models. This ratio is probably significant in the modulation that would be excited by a combination of first harmonic and fundamental excitation. A ratio of 0.66 , for example, would lead to a modulation every second fundamental period, as found in the model, if a mode combining the first harmonic and fundamental were excited. 
It is known observationally (Arp 1955) that the longer period Pop II Cepheids demonstrate irregularity in oscillation simflar to that of RV Tauri variables. The fact that this irregularity, in the form of an alternation of maxima of successive cycles, appeared in this model, suggests that the model is a qualitatively correct representation of the real pop II Cepheids. Since the principal peculiarity of the model was Its very large luminosity to mass ratio, I conclude that it is probably this property (a very low value of $\mathrm{g}$ ) that distinguishes the pop II Cepheids and also the RV Tauri varlables. The small metal content of the model is probably unimportant in its direct effect on the pulsation but is of prime importance in determining the evolutionary history of the model and thereby its large $L / M$. It would be necessary to study a serles of models of varying $L$ and $M$ as was done in the study of RR Lyrae pulsation (Christy 1966) in order to refine the conclusions about the Iuminosity and mass of these variables.

The pulsation was particularly interesting to investigate in detail because of its remarkable violence. At full amplitude, the photosphere varled in depth from $60 \mathrm{gm} / \mathrm{cm}^{2}$ at maximum light to $1000 \mathrm{gm} / \mathrm{cm}^{2}$ at minimum light. The material that was in the photosphere near minimum light was heated to $45,000^{\circ} \mathrm{K}$ at maximum compression. The shock in the photosphere was very strong with a shock velocity of about $19 \mathrm{~km} / \mathrm{sec}$ and a compression ratio of about 16 . However, these results are not reliable since the optically thin regions are allowed to radiate strongly in the diffusion treatment used. This resulted in an almost isothermal shock in the photosphere. 
Because of the very great variation in depth of the photosphere, the behavior in time of a fixed mass layer is significantly different from that of a fixed optical depth. The time dependence of the velocity at an optical depth of 0.1 and the radius of the photosphere are shown in Figure 3. Both of these show the alternation from one cycle to the nert. Finally, the succession of shocks appeared to be driving the topmost zone steadily away from the star. This corresponds to a rate of mass loss of $5 \times 10^{-6} \mathrm{M}$ per year and implies a dissipation of kinetic energy into potential energy at a rate of $2 \times 10^{-3}$ of the luminous $f$ lux. This is small compared to the total rate of generation of mechanical energy which is 7.2 percent of the luminous flux. Again, however, although the possibility of mass loss is clearly suggested by this result, the methods used in the numerical work preclude reliable conclusions on the behavior of the optically very thin regions involved. The motion of zones 38 (the outermost), 37, 36, and 34 is shown in Figure $1 \mathrm{~b}$.

Th1s work has been supported in part by the office of Naval Research Contract [Nonr-220(47)] and the National Aeronautics and Space Administration Grant [NsG-426].

ROBERT F. CHRISTY

January , 1966

California Institute of Technology

Pasadena, California 


\section{REFERENCES}

Abt, H. A. 1954, Ap. J. Supp. 1, 63.

Arp, H. C. 1955, Ap. J. 60, 1.

Christy, R. F. 1964, Rev. Mod. Phys. 36, 555.

Christy, R. F. 1966, Ap. J.

Schwarzschild, M. 1941, Ap. J. $94,245$.

\section{FIGURE CAPTIONS}

Figure 1. (a) The initial growth of the motion in the calculated model, shown by the radius of mass zone 34 .

(b) The ultimate behavior of the radil of the outer mass zones, showing the ejection of zone 38 .

Figure 2. The final behavior of the luminosity showing the alternation of successive periods.

Figure 3. The time behavior of the photosphere radius and of the veloclty, showing the alternation of successive periods. 

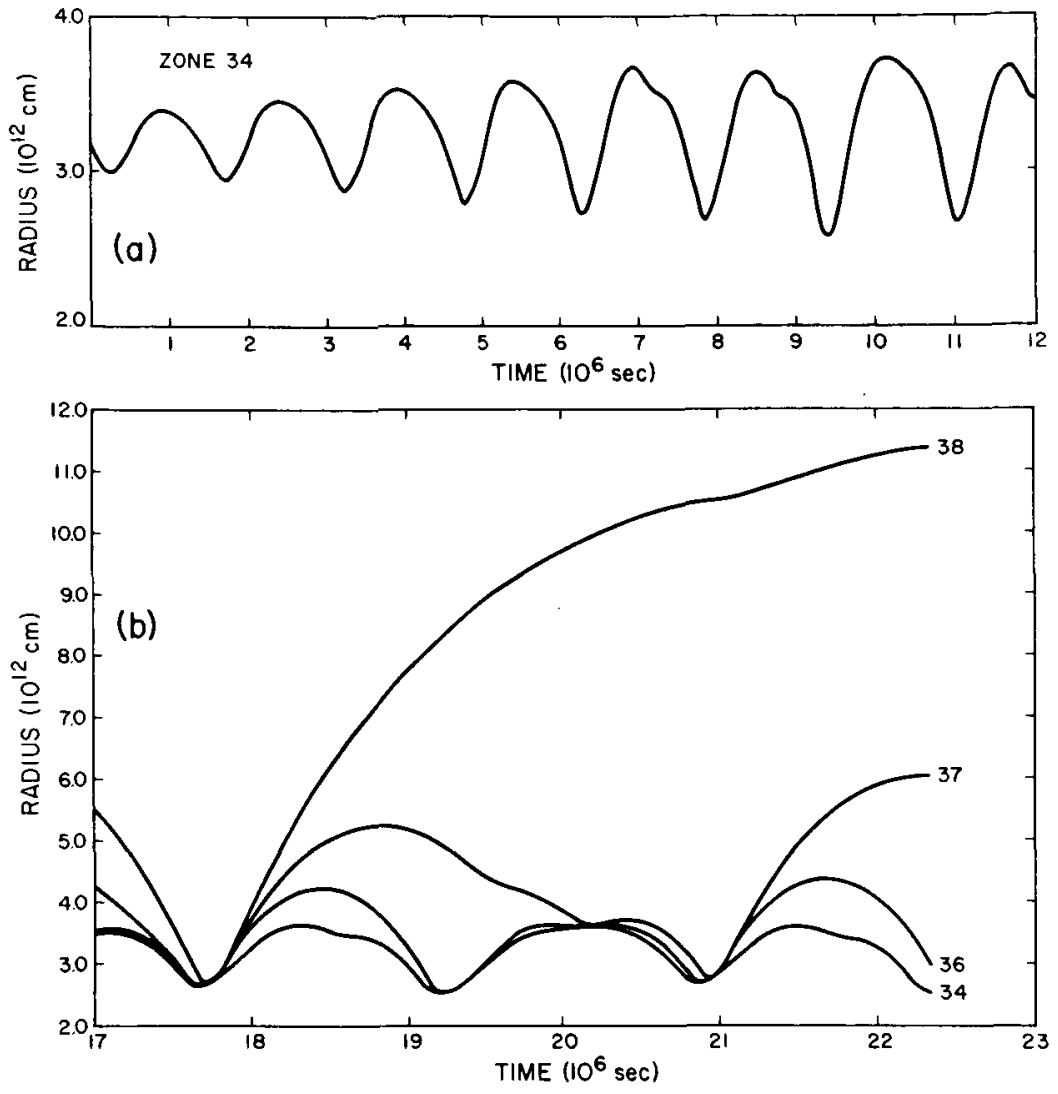

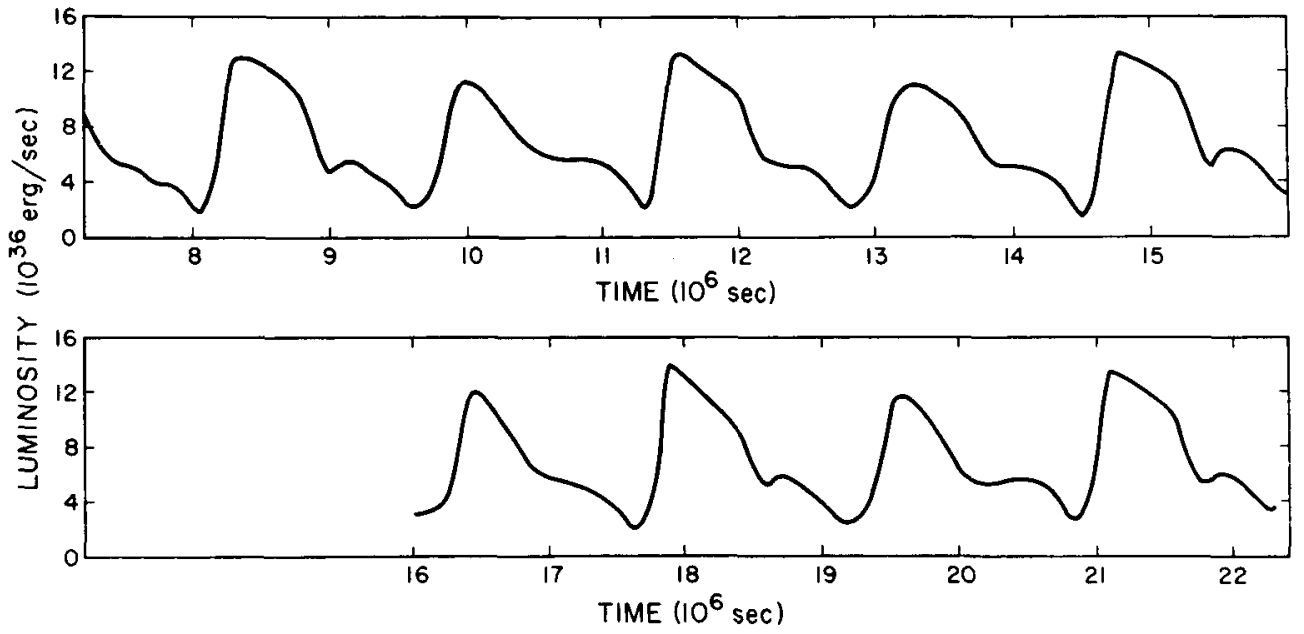

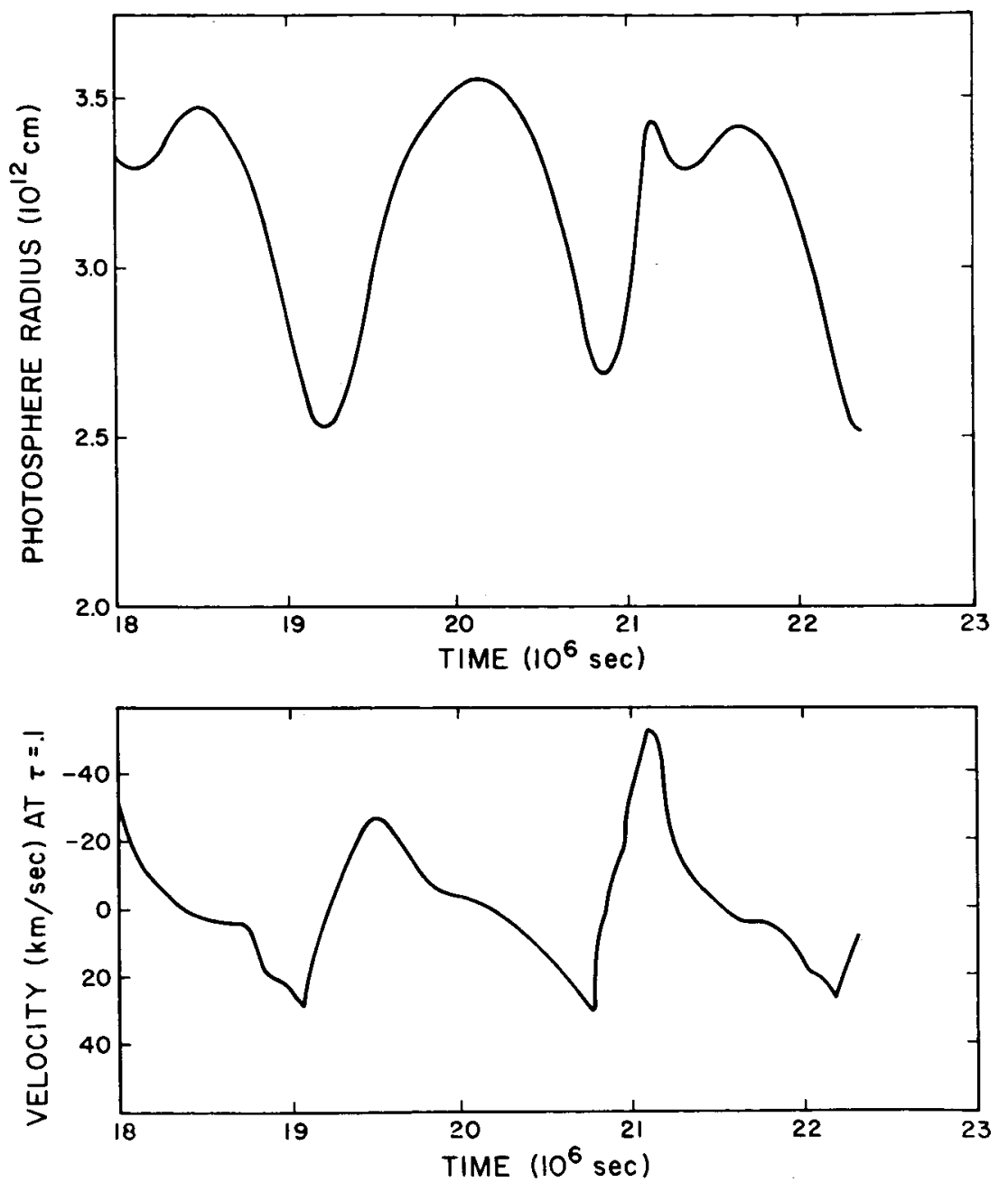